\title{
Hitting the bullseye with a nonlethal payload: resistance in CD123-positive malignancies
}

\author{
Lukasz P. Gondek
}

Johns Hopkins University School of Medicine, Department of Oncology, Division of Hematologic Malignancies, Baltimore, Maryland, USA

\begin{abstract}
The interleukin 3 receptor (CD123) is a transmembrane protein that is absent or hardly expressed on normal hematopoietic stem cells, but highly expressed on the surface of cancer cells in several hematologic malignancies. In this issue of the $J C I$, Togami et al. investigated the mechanism of resistance to the recently approved anti-CD123 agent tagraxofusp, which consists of interleukin 3 fused to a truncated diphtheria toxin (DT) molecule. The authors demonstrated that loss of the intracellular target for DT, diphthamide, a conservative modification of histidine 715 in eukaryotic elongation factor $\mathbf{2}$, resulted in tagraxofusp resistance. Specifically, hypermethylation of the DPH1 gene, encoding a key enzyme in diphthamide synthesis, resulted in diphthamide loss. Notably, treatment with a DNA hypomethylating agent restored $D P H 1$ expression and resensitized cells to tagraxofusp. The recognition of this resistance mechanism may have important clinical implications and lead to the development of more effective multiagent therapies.
\end{abstract}

\section{Searching for an ideal cancer- specific "target"}

Monoclonal antibodies, introduced to the oncology armamentarium over 2 decades ago, initiated a golden era of targeted cancer immunotherapy. A plethora of therapies, including unconjugated antibodies, antibody-drug conjugates (ADCs), bispecific $\mathrm{T}$ cell engagers (BiTEs), or more recently, chimeric antigen receptor (CAR) $\mathrm{T}$ cells, have been approved for various hematologic malignancies. However, the safety and efficacy of targeted immunotherapy rely heavily on the nature of the target antigen. The ideal "target" should be abundantly and exclusively expressed on the surface of cancer cells, and minimally secreted to maximize cancer cell binding. Additionally, depending on the desired mechanism of action, it should either internalize rapidly to deliver a cytotoxic conjugate intercellularly, or internal- ize minimally to induce complement- or antibody-dependent cytotoxicity. Moreover, an ideal "target" ought to be vital for cancer cell survival to minimize the emergence of target-negative clones and secondary resistance. Unfortunately, there are only a few cell-surface antigens that fulfill the aforementioned criteria.

CD123 is an $\alpha$ subunit of the interleukin 3 receptor (IL-3R) that functions as a heterodimer composed of $\alpha$ and $\beta$ chains. IL-3 is a pleiotropic cytokine produced by $\mathrm{T}$ cells that is crucial for hematopoietic stem and progenitor cell (HSPC) and endothelial cell proliferation and differentiation (1). Although CD123 is absent or expressed at low levels on normal hematopoietic stem cells (HSCs), its cell-surface density is higher in myeloid and B lymphocyte progenitors (2). As opposed to healthy HSPCs, CD123 is highly expressed on the surface of cancer

Related Article: p. 5005

Conflict of interest: The author has declared that no conflict of interest exists.

Copyright: @ 2019, American Society for Clinical Investigation.

Reference information: / Clin Invest. 2019;129(11):4590-4592. https://doi.org/10.1172/JCI132443.

cells in several hematologic malignancies including blastic plasmacytoid dendritic cell neoplasm (BPDCN) $(3,4)$, hairy cell leukemia (5), acute myeloid leukemia (AML) (6), and B cell acute lymphoblastic leukemia (7), making this molecule a promising therapeutic target.

\section{Hitting the bullseye on cancer cells (with a ricochet)}

In December 2018, the Food and Drug Administration approved tagraxofusperzs, a human recombinant IL-3 fused to the catalytic and translocation domains of diphtheria toxin (DT), for the treatment of BPDCN. This approval was based on a pivotal, multicenter, single-agent clinical trial in patients with newly diagnosed and relapsed/refractory BPDCN. Among previously untreated patients, 90\% responded and $72 \%$ achieved complete response. The overall response rate was $67 \%$ in relapsed refractory patients (8). This is the first drug approved for BPDCN and as a single agent constitutes a favorable alternative to an intensive multiagent chemotherapy. Given the presence of CD123 on HSPCs and endothelial cells, capillary leak syndrome and cytopenias were among the most frequent on-target side effects.

Unfortunately, a subset of cancers displays primary resistance to tagraxofusp or acquired secondary resistance after achieving clinical response. Until now, the mechanism of resistance has remained largely unknown.

\section{Overcoming drug resistance}

In order for tagraxofusp to impose its antitumor effect, both IL-3 and truncated DT need to work flawlessly. The IL-3 portion binds to IL-3R, enabling target acquisition. Subsequently, the complex is internalized and acidified. The truncated DT is then translocated into the cytoplasm where it binds to eukaryotic elongation factor 2 (eEF2) and catalyzes ADP-ribosylation of posttranslationally modified histidine, called diphthamide, in eEF2. This mod- 
A

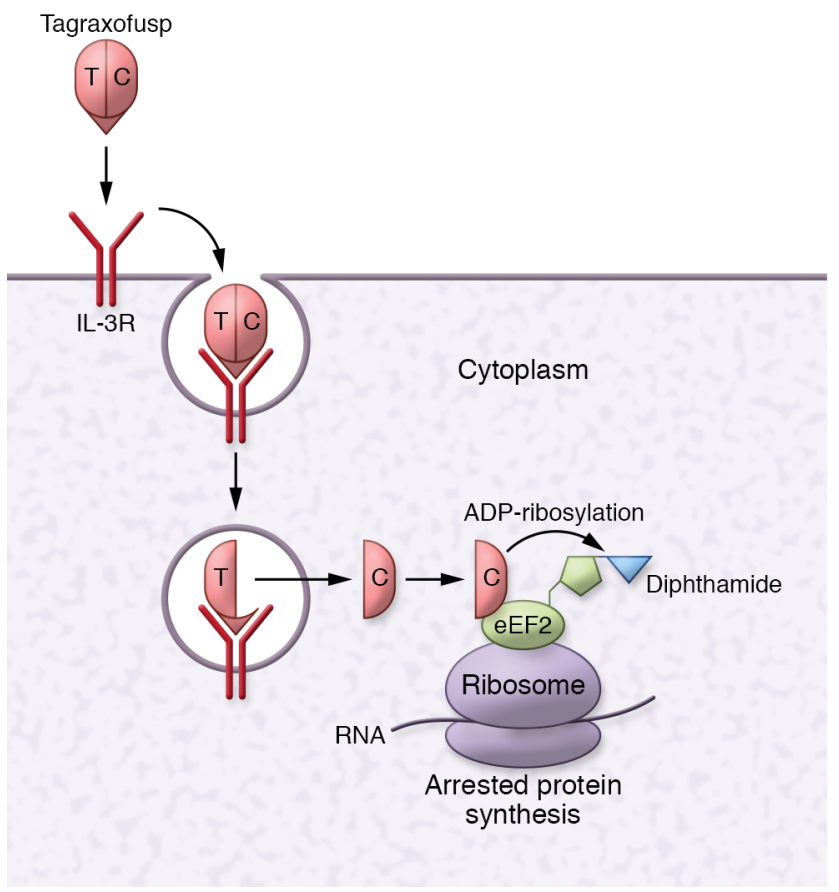

B

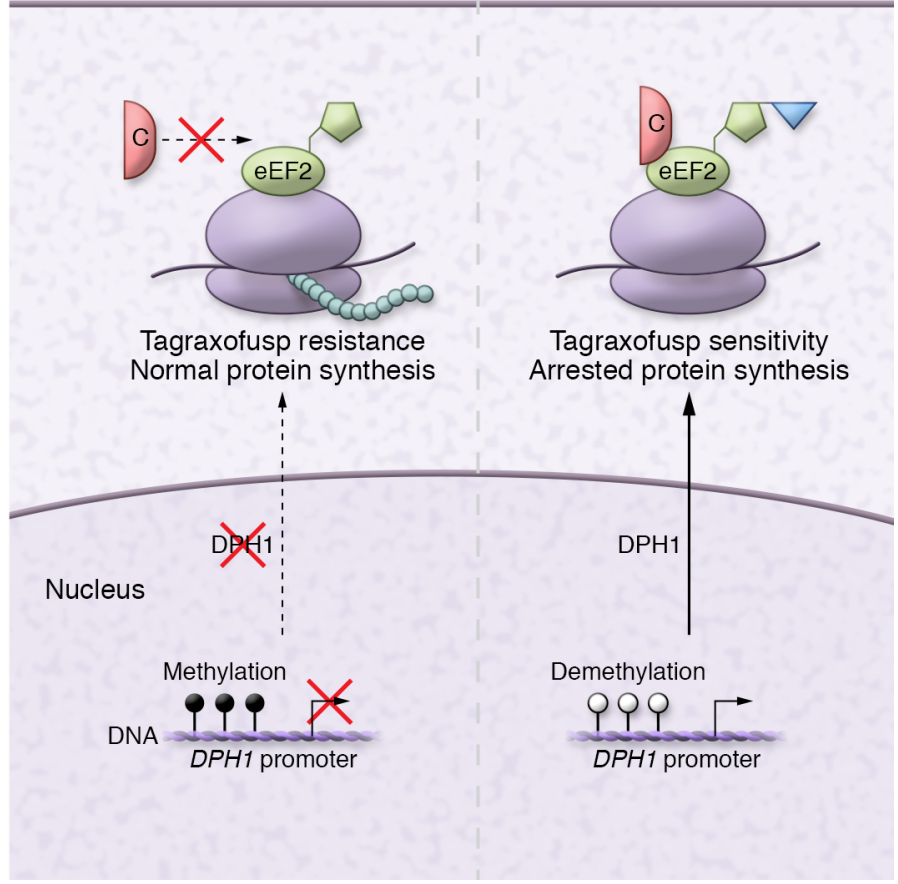

Figure 1. Mechanism of action and resistance to tagraxofusp. (A) Tagraxofusp-mediated cell killing includes IL-3R engagement, endocytosis, acidification, and translocation of DT into the cytoplasm; ADP-ribosylation of diphthamide on eEF2, and inhibition of protein synthesis. (B) Model showing that hypermethylation of the $D P H 1$ promoter prevents diphthamide modification of eEF2 and confers tagraxofusp resistance, while demethylation of the $D P H 1$ promoter restores diphthamide synthesis and sensitivity. T, translocation domain; C, catalytic domain.

ification halts protein synthesis and kills the cell (Figure 1A). Thus, the resistance to tagraxofusp may arise at any level of this multistep process.

In this issue of the JCI, Togami et al. elegantly investigated the complex nature of tagraxofusp resistance in a logical stepby-step approach. Using primary human and in vitro cell models, the authors demonstrated that CD123 expression is vital for cancer cell survival, and that resistance to tagraxofusp is not coupled with CD123 loss (9). Loss-of-target resistance has been previously observed in lymphoid malignancies during anti-CD19 targeted therapies, suggesting that unlike CD123, CD19 is not necessary for leukemia survival (10). Thus, in this regard, CD123 appears superior as a cell-surface target.

After excluding target engagement as a source of resistance, Togami and colleagues focused on the DT component and discovered that tagraxofusp-resistant cancer cells were in fact resistant to DT. To further examine the mechanism, the authors performed a whole-transcriptome RNA profiling and found that the diphthamide biosynthesis 1 (DPH1) gene was the most downregulated in resistant cells (9). DPH1 encodes the first of 7 enzymes (DPH1-DPH7) in the synthesis pathway of diphthamide, a conserved modification of histidine 715 in eEF2 (Figure 1B). Togami et al. elegantly confirmed that DHP1 loss of function resulted in tagraxofusp resistance using unbiased genome-wide screens. Next, the authors demonstrated that decreased DPH1 expression was likely due to hypermethylation of its promoter; treatment with a hypomethylating agent, azacitidine, restored the DPH1 expression and resensitized cells to tagraxofusp (Figure $1 \mathrm{~B})$. These findings were neatly confirmed using patient-derived BPDCN xenografts in mice (9).

\section{Conclusions and future directions}

Proper insight into the mechanism of tagraxofusp resistance may have several important clinical implications: (a) CD123 is a promising target given its fundamental role in cancer survival. Because the resistance to anti-CD123 therapy is likely unrelated to target loss, cross-resistance to other anti-CD123 agents currently in development (CAR-T cells, CD123-CD3 BiTEs or DARTs) should not be of major clinical concern. (b) Loss of diphthamide synthesis may result in resistance to other ADCs containing DT or pseudomonal toxin. (c) Hypomethylating agents may "resensitize" cancer cells and act synergistically with tagraxofusp. At least in some instances, DT resistance arises due to epigenetic silencing of the DPH1 locus. In fact, a multicenter phase 1 clinical trial was recently launched to examine the efficacy of the combination of azacitidine and tagraxofusp (NCT03113643).

The findings of Togami and colleagues may result in more effective multiagent therapies. However, several clinical considerations need addressing: (a) Selective pressure applied through therapies may result in either expansion of primary resistant clones or acquisition of secondary resistance in previously sensitive cells. It is unclear whether the former, the latter, or both contribute to tagraxofusp resistance in BPDCN and AML. Future experiments to investigate these resistance mechanisms are needed and will influence our 
therapeutic approaches. (b) There are currently no biomarkers of response to tagraxofusp. Current data suggest that tagraxofusp-dependent ADP-ribosylation may distinguish sensitive from primary resistant tumors, making it a promising marker of response. However, prospective clinical trials are needed to validate ADP-ribosylation as a diagnostic marker. (c) Little is known about the role of diphthamide outside of DT-mediated cell death. There is a growing body of evidence pointing toward its role in mRNA translation fidelity, cell survival, and oncogenesis $(11,12)$. It is not surprising that this highly conserved eEF2 modification evolved to serve beyond acting as a substrate for DT-mediated cell killing, but also serves to control fundamental cellular functions, such as accurate protein synthesis. Further studies should investigate significant, yet undescribed functions of eEF2 diphthamide modification.

\section{Acknowledgments}

The author acknowledges support from the NIH (grants K08HL136894 and R21HL143096).

1. Testa U, Pelosi E, Frankel A. CD 123 is a membrane biomarker and a therapeutic target in hematologic malignancies. Biomark Res. 2014;2(1):4

2. Huang S, et al. Correlation between IL-3 receptor expression and growth potential of human CD $34^{+}$hematopoietic cells from different tissues. Stem Cells. 1999;17(5):265-272.

3. Garnache-Ottou F, et al. Extended diagnostic criteria for plasmacytoid dendritic cell leukaemia. Br J Haematol. 2009;145(5):624-636.

4. Chaperot L, et al. Identification of a leukemic counterpart of the plasmacytoid dendritic cells. Blood. 2001;97(10):3210-3217.

5. Del Giudice I, et al. The diagnostic value of CD123 in B-cell disorders with hairy or villous lymphocytes. Haematologica. 2004;89(3):303-308.

6. Jordan CT, et al. The interleukin-3 receptor alpha chain is a unique marker for human acute myelogenous leukemia stem cells. Leukemia.
2000;14(10):1777-1784.

7. Muñoz L, et al. Interleukin-3 receptor alpha chain (CD123) is widely expressed in hematologic malignancies. Haematologica. 2001;86(12):1261-1269.

8. Pemmaraju N, et al. Tagraxofusp in blastic plasmacytoid dendritic-cell neoplasm. N Engl JMed. 2019;380(17):1628-1637.

9. Togami K, et al. DNA methyltransferase inhibition overcomes diphthamide pathway deficiencies underlying CD123targeted treatment resistance. J Clin Invest. 2019;129(11):5005-5019.

10. Sotillo E, et al. Convergence of acquired mutations and alternative splicing of CD19 enables resistance to CART-19 immunotherapy. Cancer Discov. 2015;5(12):1282-1295.

11. Pellegrino S, et al. Structural insights into the role of diphthamide on elongation factor 2 in mRNA reading-frame maintenance. J Mol Biol. 2018;430(17):2677-2687.

12. Stahl S, et al. Loss of diphthamide pre-activates NF- $\mathrm{NB}$ and death receptor pathways and renders MCF7 cells hypersensitive to tumor necrosis factor. Proc Natl Acad Sci U S A. 2015;112(34):10732-10737. 